\title{
Cirugía conservadora del angiomiolipoma renal bilateral durante el embarazo
}

\author{
Gimeno Argente V, Bosquet Sanz M, Bonillo García MA, Gómez Pérez L, Pontones Moreno JL, \\ Jiménez Cruz JF.
}

Servicio de Urología. Hospital Universitario La Fe. Valencia.

Actas Urol Esp 2006; 30 (6): 633-637

\section{RESUMEN}

CIRUGÍA CONSERVADORA DEL ANGIOMIOLIPOMA RENAL BILATERAL DURANTE EL EMBARAZO

La existencia de angiomiolipomas (AML) renales bilaterales es relativamente infrecuente, sobre todo cuando no se presentan asociados a síndromes como la esclerosis tuberosa o la linfangioleiomiomatosis. Presentamos el caso de una mujer de 40 años que durante la $33^{a}$ semana de gestación fue diagnosticada, de forma incidental en una ecografia abdominal de control, de AML renales bilaterales y que pudo ser tratada mediante cirugía conservadora del parénquima renal. Además realizamos una revisión de la literatura, centrándonos en el manejo de esta entidad y su relación con el embarazo.

Palabras clave: Angiomiolipoma renal bilateral. Embarazo. Cirugía conservadora.

\section{ABSTRACT}

\section{CONSERVATIVE SURGERY OF BILATERAL RENAL ANGIOMYOLIPOMA DURING PREGNANCY}

The existence of bilateral renal angiomyolipoma (AML) is fairly infrequent, especially when not associated with such syndromes as tuberous sclerosis or linfangioleimiomatosis. Here we present the case of a 40-year-old woman who was accidentally diagnosed at week $33^{\text {rd }}$ of gestation by an ultrasound, of bilateral renal AML and that could be treated with kidney sparing conservative treatment. We have also done a review of the literature focusing on its management and its relationship with pregnancy.

Keywords: Bilateral renal angiomyolipoma. Pregnancy. Conservative surgery.

$\mathrm{E}^{1}$ angiomiolipoma renal (AML) es un tumor benigno relativamente infrecuente que aparece en el 0,3\% de la población general y supone el $3 \%$ de las masas renales sólidas ${ }^{1-3}$. Está constituido, en proporción variable, por tres estirpes celulares: tejido adiposo maduro, músculo liso y vasos sanguíneos irregulares. Habitualmente, se presenta bajo dos formas clínicas: como un tumor único, grande, en mujeres de mediana edad (el 80\% de todos los AML), o bien asociado a la enfermedad de Bourneville o esclerosis tuberosa, donde suele ser bilateral, múltiple, de pequeño tamaño y con mayor incidencia en varones jóvenes ${ }^{4}$.
La asociación de AML y embarazo, aunque rara, se ha descrito previamente en la literatura ${ }^{5-9}$. Presentamos un caso donde coexisten ambas entidades, lo que lo hace más extraordinario, a la vez que plantea varias incógnitas en cuanto a su manejo, no siempre fáciles de resolver.

\section{CASO CLÍNICO}

Presentamos el caso de una mujer de 40 años de edad, sin antecedentes medico-quirúrgicos de interés, primigesta con embarazo gemelar de 33 semanas. Durante el ingreso en el Servicio de Ginecología de nuestro hospital, en el contexto del estudio ecográfico de una colestasis intrahe- 
pática gestacional, se detecta de forma incidental la presencia de una masa renal bilateral. Dado que la paciente se encuentra asintomática, estable hemodinamicamente y con una exploración física y analitica dentro de la normalidad, se decide una actitud expectante.

A los 3 días presenta un episodio de bradicardia fetal que motiva la realización de una cesárea urgente bajo anestesia locorregional, que transcurre sin incidencias. Inmediatamente tras la cesárea se practica TC abdómino-pélvico para filiar las masas renales, donde se objetiva una hemorragia retroperitoneal con un hematoma perirrenal derecho y otro intrarrenal izquierdo (Fig. 1). La enferma permanecía estable sin referir dolor lumbar, hematuria ni otros síntomas urológicos. A la exploración física presenta buen estado general, temperatura axilar de $37,2{ }^{\circ} \mathrm{C}$, presión arterial de 130/80 mmHg y frecuencia cardiaca de 82 lat/min. En la palpación abdominal no se detectan masas. Los datos analíticos sanguíneos son normales salvo una hemoglobina de 7,4 g/dl y un hematocrito de $23,7 \%$, que requiere la transfusión de 2 unidades de concentrado de hematíes. Se practica una urorresonancia magnética de control, que evidencia un hematoma perirrenal bilateral con desplazamiento anterior de estructuras intraabdominales, así como una masa en la porción posterior del polo superior del riñón derecho, de tamaño aproximado de $7 \times 5 \times 4 \mathrm{~cm}$, bien delimitada, y otra en el tercio medio del riñón izquierdo, de $2 \times 3 \mathrm{~cm}$, con signos de sangrado reciente (Figs. 2a, 2b y 2c).

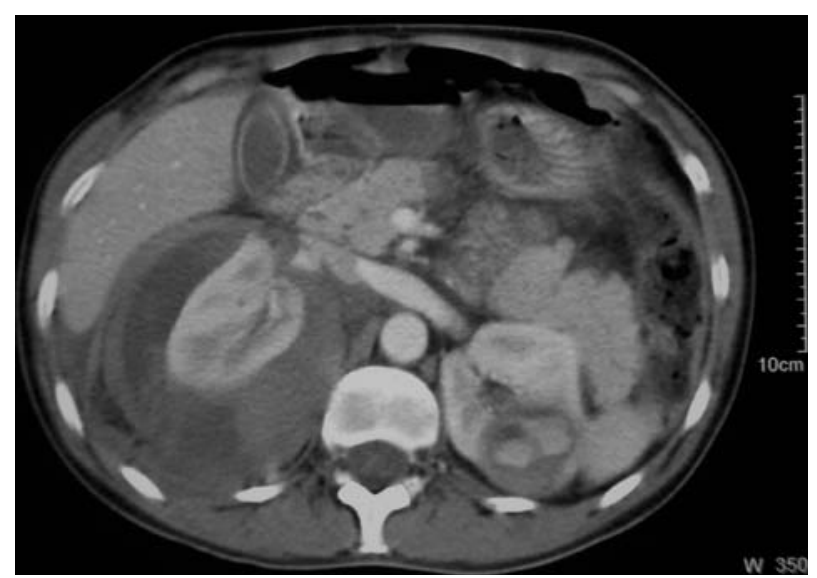

FIGURA 1: Imagen de TC abdomino-pélvico con contraste que muestra una colección hemática en espacio perirrenal derecho, con desestructuración del polo superior renal $y$ hematoma intrarrenal izquierdo.
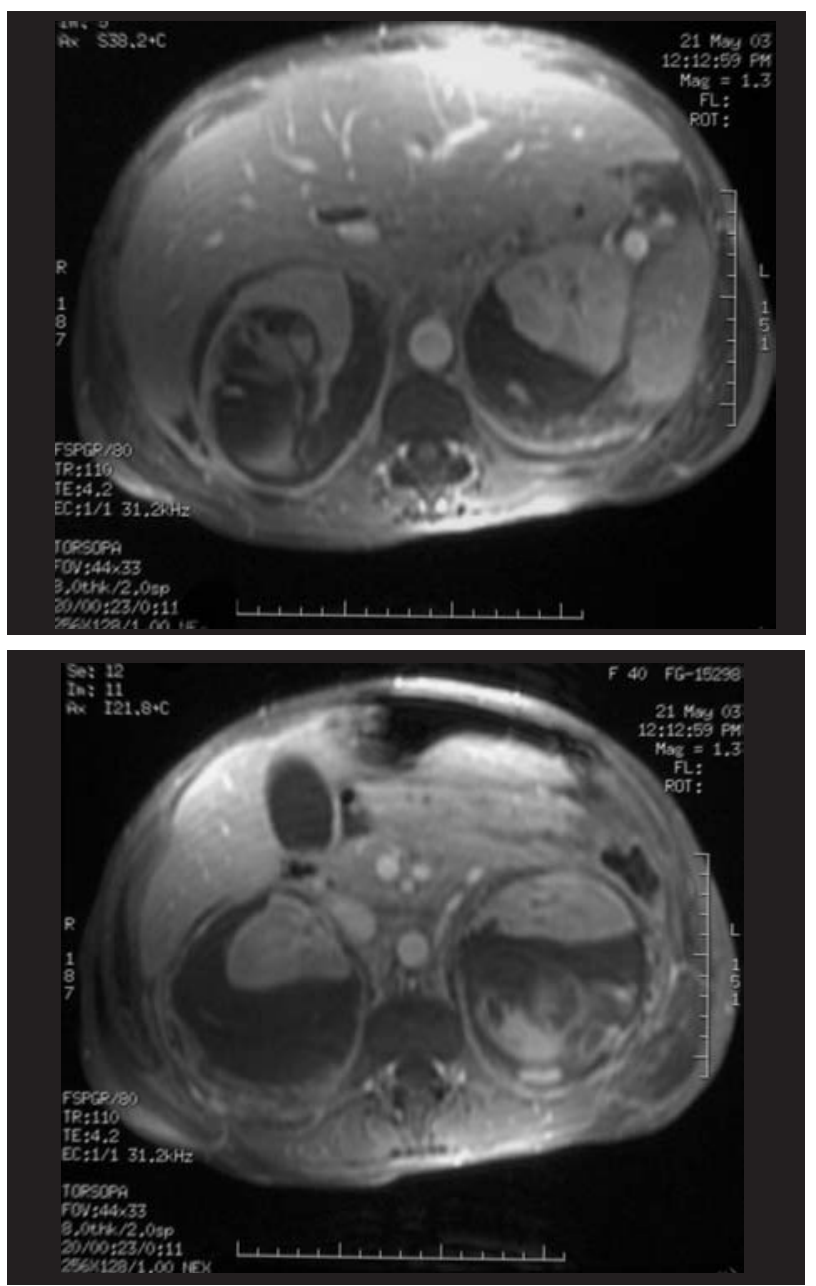

FIGURAS $2 a$ y 2b: Imágenes de RM abdominal donde se observa un hematoma perirrenal bilateral, asi como una masa hipointensa en polo superior de riñón derecho, de tamaño aproximado $7 \times 5 \times 4 \mathrm{~cm}$., junto a otra masa situada en tercio medio renal izquierda, de $2 \times 3 \mathrm{~cm}$.

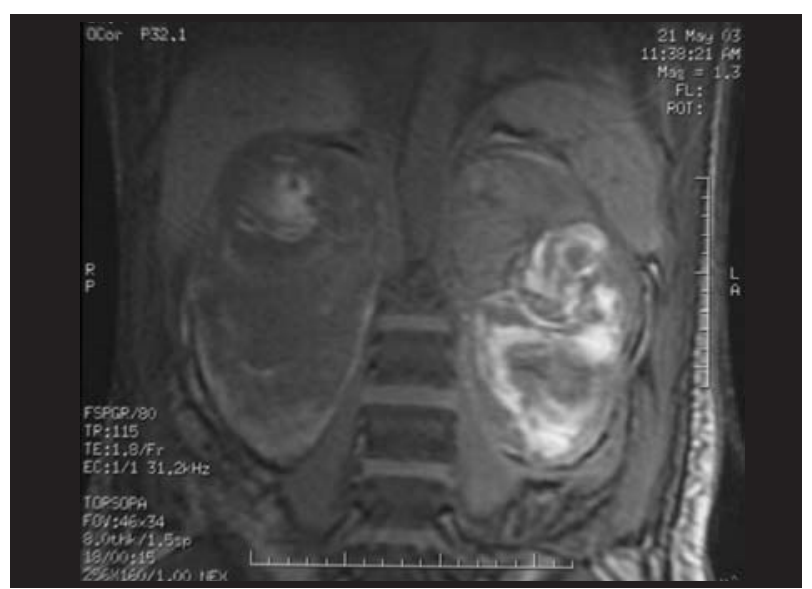

FIGURA 2c: Corte coronal de RM abdominal donde se aprecia la existencia de masas renales bilaterales, con signos de sangrado en varios tiempos (las áreas hiperintensas del riñón izquierdo, corresponden a hemorragia aguda procedente de la masa heterogénea situada en tercio medio renal). 
Durante las siguientes 48 horas continua descendiendo el hematocrito pese a repetidas transfusiones sanguíneas, apareciendo de forma súbita un cuadro de dolor lumbar intenso, malestar general, hipotensión arterial (90/50 $\mathrm{mmHg})$ y caída brusca del hematocrito $(17,2 \%)$. Ante tales hallazgos y dada la inestabilidad hemodinámica de la paciente, se decide la intervención quirúrgica urgente, practicando una incisión subcostal bilateral (Chevron), evacuando el contenido hemorrágico acumulado en el espacio retroperitoneal y objetivando las citadas masas renales, de las que se toman biopsia extemporánea intraoperatoria. Se procede a la exploración del tumor renal derecho que ocupa el polo superior renal, practicándose una nefrectomía polar. A continuación se examina la masa renal izquierda, realizándose una tumorectomía con posterior nefrorrafia.

El estudio histológico de las masas extirpadas, pone de manifiesto la presencia de unas lesiones constituidas por tejido adiposo maduro en cuyo seno se encuentran numerosas estructuras vasculares sanguíneas, provistas en general de capas musculares lisas y de luces dilatadas, junto con áreas de tejido muscular liso con acúmulos de histiocitos y lipófagos (Figs. 3a y 3b), con reactividad inmunohistoquímica frente a HMB-45 y MART-1, mostrando extensas áreas de infiltración hemorrágica con extensión a grasa perirrenal. Estos hallazgos son compatibles con el diagnóstico anatomopatológico de angiomiolipoma renal.
La enferma presenta un postoperatorio sin incidencias, con evolución satisfactoria y alta hospitalaria a las 2 semanas. Se realiza TC abdómino-pélvico a los 3 meses de la cirugía, demostrando la indemnidad del parénquima renal y la reabsorción del hematoma perirrenal derecho. Tras 15 meses de evolución, la paciente se encuentra asintomática con preservación de la función renal.

\section{DISCUSIÓN}

Los angiomiolipomas renales bilaterales aparecen hasta en un $80 \%$ de pacientes afectos de esclerosis tuberosa, siendo múltiples y de pequeño tamaño ${ }^{3,10}$. Cuando aparecen de forma esporádica, estos tumores suelen ser únicos, de gran tamaño y asintomáticos ${ }^{11,12}$.

En nuestro caso, destacamos la bilateralidad de los angiomiolipomas, sin que exista asociación con esclerosis tuberosa ni otras enfermedades afines como la linfangioleiomiomatosis. Con esta entidad, con la que se asocia hasta en un $57 \%$ de ocasiones, comparte ciertas características comunes como son la presencia de receptores estrogénicos y progesterónicos en las células musculares lisas y la positividad a la inmunorreacción HMB-45, provocada por la existencia de anticuerpos monoclonales que reaccionan específicamente con premelanosomas presentes en las células musculares lisas ${ }^{8,13}$. La importancia de esta reactividad inmunohistoquímica radica en su capacidad para diferenciar el angiomiolipo-

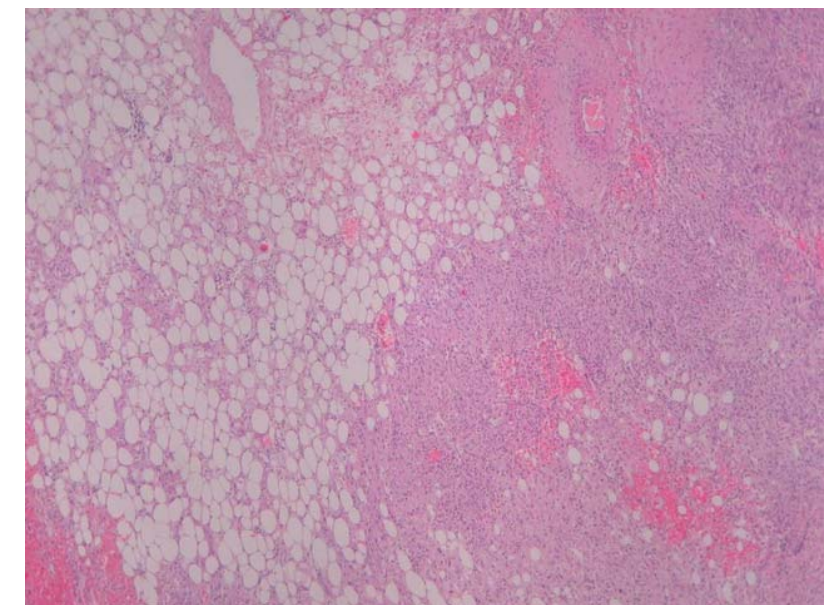

FIGURAS $3 a$ y 3b: Imagen microscópica de anatomía patológica que muestra lesiones proliferativas tumorales implantadas sobre el parénquima renal, constituidas por una proliferación de células fusiformes entremezcladas con áreas de diferenciación adipocitaria y gran cantidad de vasos sanguíneos con cambios parietales mixoides. 
ma de otros tumores derivados del tejido conectivo como el liposarcoma, el histiocitoma fibroso maligno, el leiomiosarcoma o el carcinoma sarcomatoide; pues es el angiomiolipoma, el único tumor renal que presenta reactividad frente a HMB-45. Esta confirmación es fundamental para determinar la naturaleza tumoral y la actitud terapéutica más adecuada para el paciente ${ }^{14,15}$.

La utilización cada vez más extendida de la ecografia y el TC como exploraciones diagnósticas de procesos abdominales, ha producido un notable incremento en el diagnóstico incidental de masas renales $^{9}$. En nuestra paciente, el hallazgo casual de estos tumores en fase asintomática, estuvo condicionado a la exploración ecográfica de control durante el periodo de gravidez. Algunos autores postulan que el angiomiolipoma renal experimenta un evidente desarrollo durante la gestación, lo que indica una clara influencia hormonal ${ }^{8}$. Esta hipótesis se vería apoyada por diversos factores como son el predominio en mujeres en edad fértil, donde suelen presentar tamaños superiores a 4 $\mathrm{cm}^{16}$, su rareza antes de la pubertad (excepto cuando se asocian a esclerosis tuberosa), y el hecho de que estos tumores tienen un mayor índice de crecimiento en mujeres que en varones, debido a la presencia de receptores de progesterona en las células musculares lisas ${ }^{17}$.

El manejo de los angiomiolipomas está ampliamente discutido en la literatura. La pauta terapeútica más aceptada es el algoritmo de Oesterling et al. ${ }^{18,19}$, basado en la presentación clínica, el tamaño y la bilateralidad del tumor. De esta forma, en tumores asintomáticos se realizarán controles periódicos con ecografía y/o TC cada seis o doce meses, según el tamaño sea mayor o menor de 4 $\mathrm{cm}$, respectivamente. En los tumores sintomáticos y/o bilaterales, se intentará una embolización arterial selectiva o cirugía renal conservadora (nefrectomía parcial o tumorectomía). La nefrectomía radical quedará reservada a aquellos casos con inestabilidad hemodinámica por sangrado incontrolable, tumores de gran tamaño, de localización central o coexistencia de carcinoma en el mismo riñón. En nuestra paciente, la evolución de la situación hemodinámica obligó a una intervención urgente, optando por una cirugía conservadora ante la bilateralidad del tumor, que pudo lograrse con buenos resultados posteriores.
En cuanto a la coexistencia de angiomiolipoma renal bilateral y embarazo, se podría plantear la incógnita de cuando elegir el momento más apropiado, dentro del periodo gestacional, para decidir la actuación quirúrgica, si ésta se considera indicada. En nuestro caso, no llegamos a plantearnos esta situación debido a que se practicó una cesárea urgente por bradicardia fetal. Sin embargo, parece evidente la necesidad de obviar la cirugía durante el proceso de organogénesis (8-10 semanas), durante el cual la tasa de malformaciones se acentúa, al igual que deberíamos valorar el aumento de la incidencia de abortos en el primer trimestre de gestación y la mayor posibilidad de partos prematuros durante el último trimestre que supondría cualquier actuación quirúrgica. Por todo ello, el segundo trimestre gestacional parece el momento idóneo para realizar la intervención quirúrgica, aunque es necesario individualizar en cada caso, si la cirugía está indicada.

En conclusión, el manejo actual del angiomiolipoma renal tiene como objetivo primordial la preservación del parénquima renal no tumoral, sobre todo en tumores de gran tamaño, múltiples, bilaterales o recurrentes. Entre las opciones terapéuticas con preservación de la función renal la tumorectomía y la nefrectomía parcial son las más empleadas; si bien, también son posibles la embolización arterial selectiva, la crioterapia o la aplicación de técnicas de ablación tumoral por calor como los ultrasonidos o la radiofrecuencia.

\section{REFERENCIAS}

1. Hadju SI, Foote Jr. FW. Angiomyolipoma of the kidney: report of 27 cases and review of the literature. J Urol. 1969;102(4):396-401.

2. Mazeman E, Wemean L, Biserte J. Renal angiomyolipoma. A report of 11 cases. Eur Urol. 1980;6(6):328-334.

3. Schneider-Monteiro ED, Lucon AM, De Figueiredo AA, Rodrigues Junior AJ, Arap S. Bilateral giant renal angiomyolipoma associated with hepatic lipoma in a patient with tuberous sclerosis. Rev Hosp Clin Fac Med Sao Paulo. 2003 Mar-Apr;58(2): 103-108.

4. Chesa Ponce N, Artiles Hernández JL, Ponce Socorro JM, Del Rosario Medina J, Castro López-Torrella V, Betancort De Leon R. Sindrome de Wunderlich como primera manifestación de un angiomiolipoma renal. Arch Esp Urol. 1995;48(3):305-308.

5. Fernández Arjona M, Mínguez R, Serrano P, Sanz J, Teba F, Peinado $\mathrm{F}$, et al. Angiomiolipoma renal de rápido crecimiento asociado a embarazo. Actas Urol Esp. 1994 JulAug; 18(7):755-757. 
6. Tanaka M, Kyo S, Inoue M, Kojima T. Conservative management and vaginal delivery following ruptured renal angiomyolipoma. Obstet Gynecol. 2001 Nov;98(5Pt2):932933.

7. Forsnes EV, Eggleston MK, Burtman M. Placental abruption and spontaneous rupture of renal angiomyolipoma in a pregnant woman with tuberous sclerosis. Obstet Gynecol. 1996 Oct;88(4 Pt 2):725.

8. Molina M, Ruipérez J, Ortega N, Parrilla P: Angiomiolipoma retroperiotoneal en una embarazada. Med Clin (Barc). 2001 Jul 7;117(5):199.

9. Pobil Moreno JL, Martínez Rodrigues J, Maestro Duran JL, Morales López A. Incidentaloma renal y embarazo. Arch Esp Urol. 1996;49(7):755-757.

10. Van Baal JG, Smits NJ, Keeman JN, Lindhout D, Verhoef $\mathrm{S}$. The evolution of renal angiomyolipoma in patients with Tuberous Sclerosis. J Urol. 1994;152(1):35-38.

11. Tongaonkar HB, Sampat MB, Dalal AV, Dandekar NP, Kulkarni JN, Kamat MR. Bilateral renal angiomyolipoma. J S Oncology 1994;57(1):65-70.

12. De Luca S, Terrone C, Rossetti R. Management of renal angiomyiolipoma: a report of 53 cases. BJU International. 1999;83(3):215-218.

13. Urban T, Lazor R, Lacronique J, Murris H, Cabrune S, Valeyre D, et al. Pulmonary lymphangioleiomyomatosis. A study of 69 patients. Medicine (Baltimore). 1999;78(5):321337.
14. Pea M, Bonetti F, Zamboni G, Martignoni G, Riva M, Colombari R, et al. Melanocyte-marker HMB-45 is regularly expressed in angiomyolipoma of the kidney. Pathology. 1991; 23(3):185-188.

15. Kaiserling E, Krober S, Xiao JC, Schaumburg-Lever G. Angiomyolipoma of the kidney. Inmunoreactivity with HMB-45. Light and electron-microscopic findings. Histopathology 1994;25(1):41-48.

16. Stillwell TJ, Gómez MR, Kelalis PP. Renal lesion in tuberous sclerosis. J Urol 1987;138(3):477-481.

17. Martignoni G, Pea M, Bonetti F. Progesterone receptors in renal angiomyolipoma. In: Tuberous sclerosis association of great 18. Britain. Bath, United Kingdom, 1995. An Meeting Bath, United Kingdom, 1995.

18. Oesterling JE, Fishman EK, Goldman SM, et al. The management of renal angiomyolipoma. J Urol. 1986;135(6): 11211124.

19. Martínez García R, Valls Blasco F, Domínguez Hinarejos C, Gallego Gómez J, Oliver Amorós FV, Jiménez Cruz JF. Diagnóstico y tratamiento del angiomiolipoma renal. Arch Esp Urol 1989;42(2):661-69.

Dr. V Gimeno Argente

Servicio de Urologia. Hospital Universitario La Fe

Avda. Campanar no 21, 46009. Valencia

e-mail: vigiar71@hotmail.com

(Trabajo recibido el 27 de julio 2005) 